\title{
LA SPECTROSCOPIE AU BOUT DES DOIGTS Le spectromètre pour smartphone
}

\author{
Thomas KUNTZEL \\ Arnaud ZOUBIR \\ Centre technologique en \\ Optique et Lasers ALPhANOV, \\ Talence, France \\ arnaud.zoubir@alphanov.com
}

La révolution des smartphones a véritablement permis la démocratisation de nombreuses technologies avancées, les rendant accessibles à chacun ; la technologie GPS historiquement réservée aux militaires en est un très bon exemple. Ce n'est donc pas surprenant que la spectroscopie bénéfice également des nombreux avantages qu'offrent les smartphones.

\section{I} a spectroscopie est une technique puissante qui permet d'étudier les rayonnements électromagnétiques émis, absorbés ou diffusés par la matière. Depuis son apparition au $17^{\mathrm{e}}$ siècle, l'enregistrement et l'analyse des différentes longueurs d'onde composant la lumière font qu'il est possible de décrire et comprendre des phénomènes physiques tels quel les raies de Fraunhofer qui étaient historiquement interprétées comme des bandes noires séparant les couleurs du spectre solaire. Au cours du $20^{\mathrm{e}}$ siècle, elle a connu un essor considérable avec l'avènement des capteurs électroniques, des lasers et des ordinateurs, faisant de la spectroscopie un outil indispensable de la recherche scientifique. Particulièrement, la spectroscopie d'absorption UV-visible-proche infrarouge (NIR en anglais) ainsi que la spectroscopie vibrationnelle, telle que la spectroscopie à Transformée de Fourier ou la spectroscopie Raman sont devenues le fer de lance de nombreux laboratoires mais également de nombreuses industries dans les domaines des traitements pharmacologiques, de la formulation chimique, de la biologie et bien d'autres.

Jusqu'à récemment, la spectroscopie restait une technologie plutôt encombrante reposant sur des équipements volumineux, complexes et souvent assez onéreux. De plus, ces technologies nécessitaient un savoir et un savoir-faire pour être utilisés de façon optimale. Des sociétés comme Ocean Optics (USA) ou Avantes (PaysBas) ont toutefois fait de sérieux progrès pour rendre cette technologie plus compacte et plus abordable mais le spectromètre classique reste un instrument de laboratoire plus qu'un outil accessible au citoyen ordinaire.

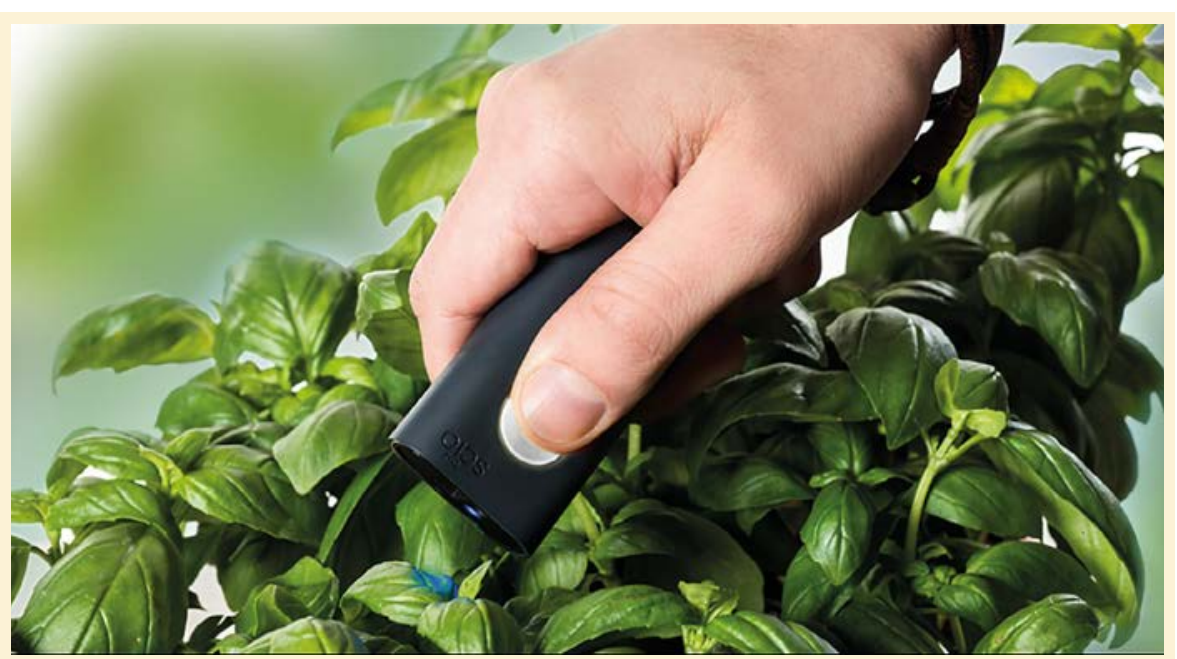

Figure 1. Capteur moléculaire SCiO de Consumer Physics.
D'autres entreprises ont récemment lancé des systèmes très compacts basés pour la plupart sur la spectroscopie NIR. Spectral Engines (Finlande) a notamment développé un appareil portatif de mesure de réflexion spectrale fonctionnant dans le NIR, idéal pour réaliser des mesures sur des lignes de production dans l'agroalimentaire ou le domaine pharmaceutique et dans de nombreux autres domaines. Consumer Physics (Israël) a récemment fait les gros titres avec un spectromètre NIR tenant dans la paume de la main et servant de capteur moléculaire, le SCiO (figure 1). Ce dispositif a pour vocation de scanner un matériau et d'obtenir instantanément des informations chimiques directement sur son smartphone (des informations nutritionnelles telles que les calories, la quantité de graisse, de glucide ou de protéines ou la composition chimique d'un médicament).

\section{Innovations et ruptures majeures}

ALPhANOV (France), un centre technologique en optique et laser, a récemment développé un dispositif universel, le GoSpectro qui s'adapte à n'importe quel smartphone et le transforme instantanément en un spectromètre diffractif opérant dans la gamme du visible de $400 \mathrm{~nm}$ à $750 \mathrm{~nm}$ avec une résolution de $10 \mathrm{~nm}$ (figure 2). Le dispositif optique composé entre autres d'une fente d'entrée, et d'un réseau de diffraction en transmission 
de 600 traits/mm est fixé à une monture s'adaptant à tout type de smartphone ou tablette numérique sous Android ou iOS. Cette monture permet également de régler et d'adapter le spectromètre à la hauteur du capteur photo. L'application mobile inclut une fonction de calibration automatique permettant une mesure précise et objective du spectre. Elle offre en outre les fonctions habituelles d'analyse de spectres comme le positionnement de curseurs, la détermination automatique de la position d'un pic, l'ajustement automatique de l'échelle du spectre ou encore la soustraction d'un spectre de référence.

De tels dispositifs peuvent aujourd'hui voir le jour grâce à la forte évolution technologique des smartphones. La plupart des smartphones disponibles sur le marché possèdent au minimum un capteur pour l'appareil photo, un microphone et pas moins d'une vingtaine de capteurs rien que pour les fonctionnalités de mouvement et de position (gyromètres et accéléromètres pour l'accélération, magnétomètres pour la fonction boussole, capteurs de gravité/pression pour l'altitude, capteur d'orientation et bien sûr le GPS) et bien d'autres encore. En combinant la puissance technologique

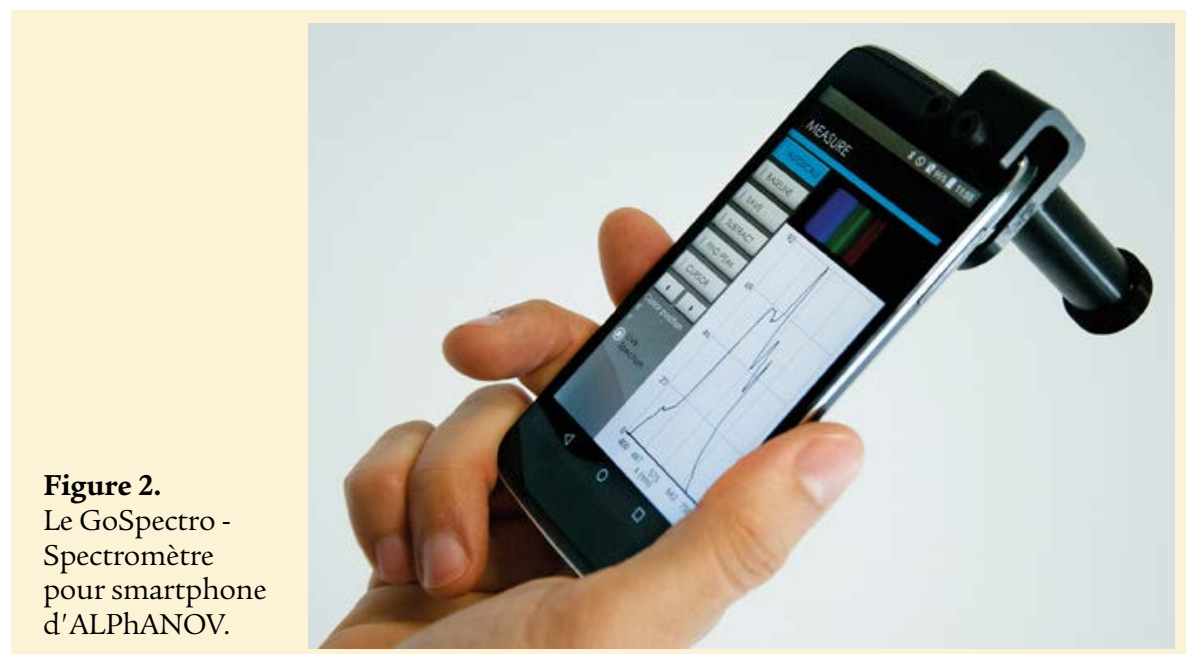

des smartphones, la miniaturisation des composants optiques (comme les réseaux de diffraction et les lentilles) et le développement mécanique et informatique, il devient alors possible de fabriquer et commercialiser un spectromètre pour smartphone à un coût abordable. Ce genre d'appareil permet de mettre la puissance de la spectroscopie entre les mains de quiconque devant réaliser des mesures de spectres lumineux sur le terrain. Le couplage avec un smartphone rend son utilisation vraiment intuitive et facile d'utilisation pour acquérir, enregistrer, exploiter et partager des spectres de lumière.

\section{Les technologies de spectrometrie pouli simartphone}

Il existe essentiellement deux méthodes pour faire de la spectrométrie visible sur smartphone: la méthode dispersive [1] et la méthode interférométrique. La méthode dispersive est basée sur la décomposition de la lumière par un prisme ou un réseau (en transmission ou en réflexion). Une image du spectre de lumière est alors obtenue et est envoyée sur le capteur du smartphone grâce à une lentille. Le capteur photo peut alors lire et enregistrer en continu des spectres. Après
Filtres Interférentiels

De 200 á 15000 nm

- Passe-bande

- Passe-haut

- Passe-bas

- Large bande

- Densité neutre

- Disponible en stock

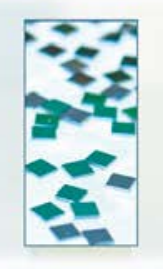

150 á 2000 nm

- Compression d'impulsion

- Télècom

- Accordabilité spectrale

- Monochromateurs

- Spectroscopie

- Disponible en stock

UK (parle francais): sales.uk@spectrogon.com·Tel +44 1592770000 US: sales.us@spectrogon.com·Tel+19733311191 
avoir correctement calibré le dispositif, il devient alors facile d'obtenir un spectre donnant l'intensité lumineuse en fonction de la longueur d'onde.

La seconde méthode utilise un interféromètre de type Mach-Zehnder (qui nécessite deux miroirs et deux lames semi-réfléchissantes) qui va générer périodiquement des interférences constructives et destructives en séparant puis recombinant le faisceau incident à l'aide des lames semi-réfléchissantes. En procédant ensuite à une transformée de Fourier, il devient possible de convertir ces retards en longueurs d'onde et donc d'obtenir un spectre. Cette méthode ne permet pas encore d'acquérir des spectres en continu du fait du temps de calcul requis pour réaliser la transformée de Fourier. En revanche, quelle que soit la méthode utilisée, la gamme spectrale d'analyse restera limitée au visible, entre $400 \mathrm{~nm}$ et $750 \mathrm{~nm}$ du fait du capteur photo du smartphone.

\section{Un peu d'histoire sur l'origine du concept}

L'idée du GoSpectro est venue après avoir constaté qu'en plaçant un dispositif optique optimisé par ALPhANOV devant le capteur photo d'un smartphone, ce dernier était en mesure de «voir» et enregistrer un spectre lumineux. Le but était alors de démontrer qu'un smartphone puisse être utilisé comme un spectromètre ayant des performances équivalentes aux produits du commerce plus coûteux. Une importante phase de développement informatique a démarré afin d'être en mesure de calibrer le plus précisément possible le

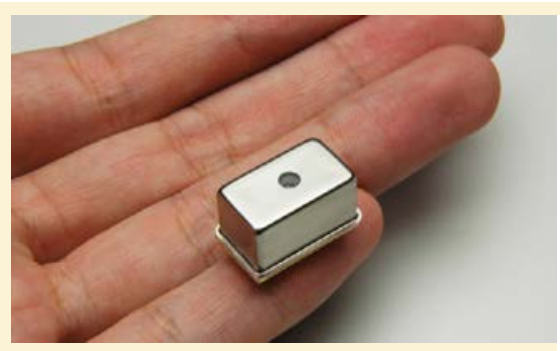

Figure 3a. Microspectromètre développé par Hamamatsu.

Figure 3b. Schéma de fonctionnement de l'OEM d'Hamamatsu. spectre de raies mesuré. Un des enjeux majeurs du développement informatique était d'intégrer ces données dans une application mobile simple, intuitive et facilement accessible. Un développement mécanique a également été nécessaire afin de rendre le dispositif compatible avec la grande majorité des smartphones. Le côté novateur et «fun» du GoSpectro a rapidement reçu un accueil positif de la communauté scientifique comme en attestent sa nomination à la vitrine de l'innovation lors de la conférence Optique Bordeaux en juillet 2016 (organisée par la revue Photoniques) et son obtention du trophée de l'innovation au cours du salon ENOVA Paris deux mois plus tard. La récente obtention du Prism Awards, la plus prestigieuse compétition internationale sur les technologies innovantes en photonique, sur le salon Photonics West à San Francisco en février 2017, a apporté une forte crédibilité et une reconnaissance sur le plan international.

\section{Évolutions techniques des spectiomètres}

L'axe majeur de développement concerne l'intégration des minispectromètres dans des dispositifs de contrôle portatifs connectés aux smartphones. Un colorimètre connecté est en cours de développement et permettra pour un château de contrôler la couleur rosée de son vin. Ce dispositif incluant une source d'excitation en entrée et le GoSpectro comme détecteur devrait permettre d'obtenir le spectre du vin, ses coordonnées colorimétriques, etc. Une autre application en cours de développement concerne l'utilisation de

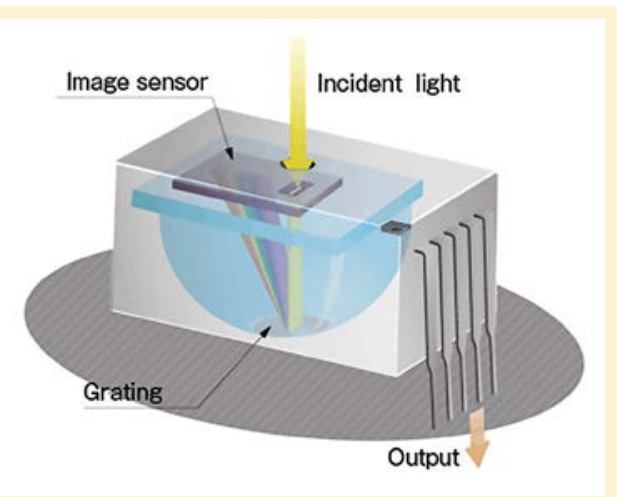

spectromètres pour smartphone dans la détection de marqueurs fluorescents. Ces dispositifs incluront un système d'excitation adapté aux fluorophores et permettront aux utilisateurs de déterminer si un produit ou un document est conforme. Notons enfin qu'il serait intéressant de pouvoir étendre dans l'UV et/ou dans l'infrarouge la plage spectrale de mesure imposée actuellement par le capteur photo du smartphone. Plusieurs solutions sont actuellement à l'étude.

Le prochain gap technologique serait d'intégrer un mini/microspectromètre directement dans un smartphone. Plusieurs entreprises sont en train de relever ce défi, notamment Hamamatsu Photonics qui a développé un OEM [2] (original equipment manufacturer) basé sur la technologie des microsystèmes électromagnétiques (MEMS) $[3,4]$. Cette tête de spectromètre, tenant sur le bout du doigt, est destinée aux instruments de mesure connectés à un smartphone ou une tablette pour des applications dans les domaines de l'inspection sanitaire, la biométrie ou le contrôle qualité de l'eau. L'ensemble $\mathrm{du}$ dispositif incluant capteur, lentilles et fentes mesure $20 \mathrm{~mm} \times 12,5 \mathrm{~mm} \times$ $10 \mathrm{~mm}$ et pèse environ $9 \mathrm{~g}$. Il opère avec une résolution typique de $10 \mathrm{~nm}$ et une plage spectrale allant de $340 \mathrm{~nm}$ à 780 $\mathrm{nm}$. Il peut être contrôlé par un carte type Arduino mais l'ultime objectif serait qu'il soit directement intégré dans un smartphone (figure 3).

\section{Marché et produits/ service dispomible}

Les spectromètres pour smartphone trouvent tout leur intérêt dans la mesure sur le terrain de spectres d'émission, de transmission, d'absorption ou de fluorescence. Parmi les principaux secteurs intéressés par la spectroscopie pour smartphone, on retrouve le domaine de l'éclairage où l'utilisation de la spectroscopie permet d'identifier instantanément le type de lampe utilisé dans les éclairages extérieurs et ce même à plusieurs mètres de hauteur. Ce genre de dispositif peut également être utilisé pour tester la conformité 


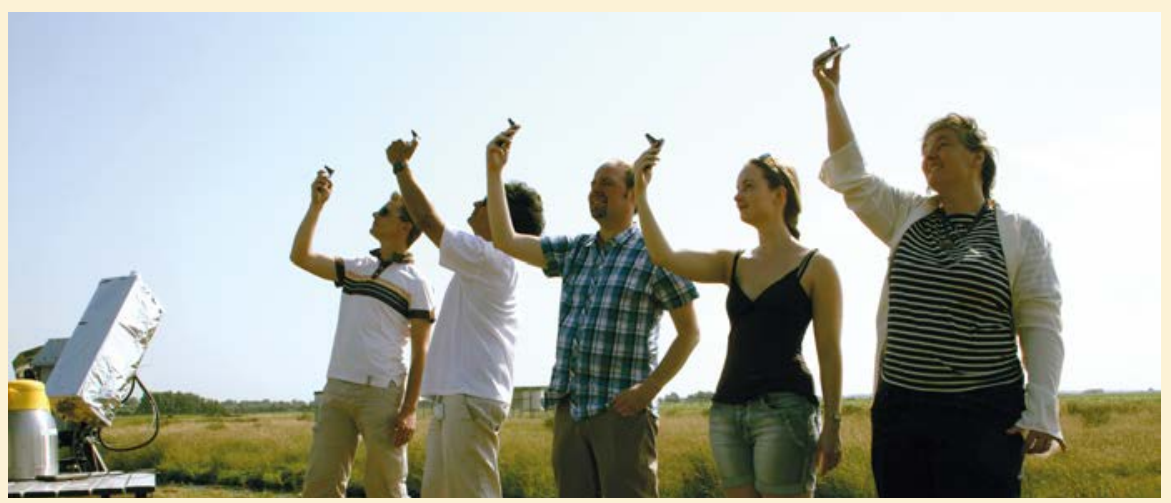

Figure 4. Photo du projet de crowdsourcing iSPEX.

du spectre d'émission d'une ampoule type LED. La traçabilité et l'authentification comme évoquées précédemment sont un autre domaine où la spectroscopie pour smartphone a tout son intérêt. Les industriels sont en effet en permanence à la recherche d'outils pouvant les aider dans leur lutte contre la contrefaçon. Avec cette technologie, vérifier l'authenticité d'un produit encore dans un camion grâce aux marqueurs fluorescents présents dans son emballage devient accessible à toute personne possédant un smartphone. La spectroscopie pour smartphone intéresse également grandement le milieu de la gemmologie, où l'étude et la caractérisation de pierres précieuses et semi-précieuses en dehors des laboratoires deviennent possibles. La facilité d'utilisation de ces appareils et leur côté ludique font que ce sont de bons outils pédagogiques pour présenter et introduire la spectroscopie à des lycéens ou étudiants.

De tels dispositifs sont en train d'ouvrir de nouvelles voies et potentiellement de nouvelles applications à la spectroscopie qui restait jusqu'à présent essentiellement réservée aux professionnels aguerris. En particulier, l'omniprésence des smartphones font d'eux de puissants outils pour collecter des grandes quantités de données. La combinaison des téléphones intelligents avec les microspectromètres a rendu possible la création d'un nouveau type de «mobile crowdsourcing» où chaque utilisateur de smartphone peut collecter des données spectrales contenant d'importantes informations. Un bon exemple de «mobile crowdsourcing» basé sur des données spectrales est présenté par le projet iSPEX financé par l'UE (http://ispex-eu.org/it). Dans ce projet, des milliers de citoyens venant des principales villes européennes ont pu mesurer la pollution de l'air grâce à un dispositif optique et une application pour smartphone dédiée fournis par les organisateurs du projet (figure 4).

Il n'est pas encore très évident d'imaginer comment cette technologie sera adoptée par les citoyens, mais avec des dispositifs qui ne nécessitent plus d'avoir un doctorat pour être utilisés, il y a de fortes chances que de nouvelles applications voient le jour. Cela se fera probablement grâce à des groupes d'amateurs de technologie, tout comme cela s'est fait avec les MEMS il y a quelques décennies, ou $l^{\prime}$ impression $3 \mathrm{D}$ plus récemment.

\section{POUR EN SAVOIR PLUS}

[1] Neece, A.G., Microspectrometers-An Industry \& Instrumentation Overview, Proc. of SPIE Vol. $7086708602-1$ (2008)

[2] Nelson, P., Texas Instruments DLP® Technology for Spectroscopy, White Paper Texas Instruments (2014)

[3] Wang, P., Review and recent progress of handheld spectrometry at Thermo Fisher Scientific, Proc. SPIE Vol. 948204-5 (2015)

[4] Antila, J., et al., MEMS- and MOEMS-Based Near-Infrared Spectrometers, Encyclopedia of Analytical Chemistry (2014)

\section{Testeur de composants}

CT440-PDL

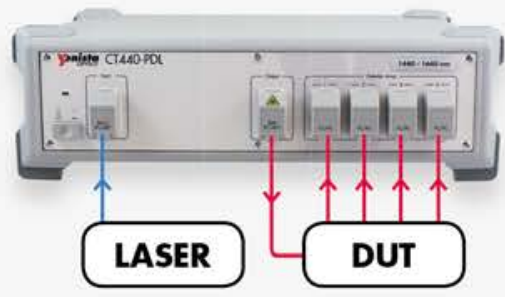

\section{Caractérisation de} composants passifs IL et PDL

\section{Modèles \\ 0 : 1260-1360 nm \\ $\mathrm{SCL}: 1440-1640 \mathrm{~nm}$}

Dynamique

$65 \mathrm{~dB}$

Précision $\lambda$ $\pm 5 \mathrm{pm}$

Précision PDL $\pm 0.05 \mathrm{~dB}+4 \%$ PDL

Résolution

5 à $250 \mathrm{pm}$

Logiciel client sur PC
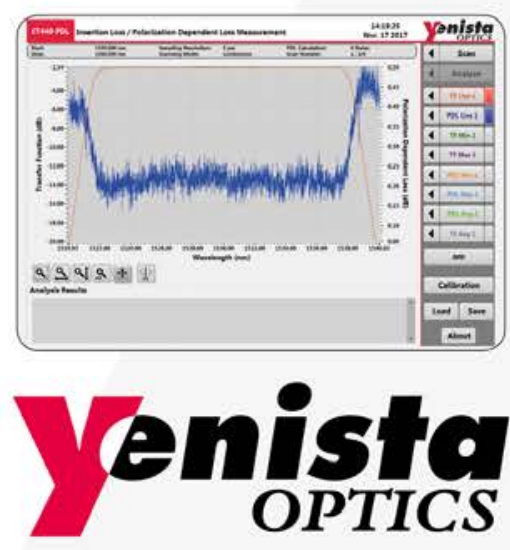

Tel.: +33 (0)2 96483716 sales-emea@yenista.com www.yenista.com 